\title{
White blood cell count is associated with features of the metabolic syndrome in European adolescents. The HELENA Study
}

\author{
F. Perez de Heredia ${ }^{1}$, L. E. Díaz ${ }^{1}$, S. Gómez-Martínez ${ }^{1}$, O. Androutsos ${ }^{2}$, J. Dallongeville ${ }^{3}$, \\ S. DeHenauw ${ }^{4}$, M. Ferrari ${ }^{5}$, L. A. Moreno ${ }^{6}$, G. Palacios ${ }^{7}$ and A. Marcos ${ }^{1}$, on behalf of the Helena \\ Study Group \\ ${ }^{1}$ Immunonutrition Research Group, ICTAN, Madrid, Spain, ${ }^{2}$ Department of Nutrition and Dietetics, Harokopio University, \\ Athens, Greece, ${ }^{3}$ INSERM U557, INRA U1125, CNAM, University Paris 13, Centre de Recherche en Nutrition Humaine, \\ 93017 Bobigny, France, ${ }^{4}$ Ghent University, Ghent, Belgium, ${ }^{5}$ Istituto Nazionale di Ricerca per gli Alimenti e la Nutrizione \\ (INRAN), Rome, Italy, ${ }^{6}$ Escuela Universitaria de Ciencias de la Salud, Universidad de Zaragoza, Zaragoza, Spain, \\ ${ }^{7}$ University Polytechnic of Madrid, Madrid, Spain
}

White blood cell count (WBCC) is used as a diagnostic tool for acute inflammatory and infectious states, but it has also been associated with other pathological conditions. In the last decade WBCC has been found to be related with obesity and the metabolic syndrome in adults $^{(1,2)}$ as well as in children and adolescents ${ }^{(3-5)}$.

The aim of the present work was to analyse the relationships between WBCC and obesity and features of the metabolic syndrome in a cohort of European adolescents participating in the HELENA-CSS (Healthy Lifestyle in Europe by Nutrition in Adolescence-CrossSectional Study).

Blood samples were collected after an overnight fast from 1,089 participants aged 12.5-17.5 years. After applying exclusion criteria (having fever and/or having received any medical treatment 24 hours prior to extraction), data were analysed from 952 adolescents (47.3\% males). The sample was divided into four categories according to WBCC quartiles. Height, weight, body mass index (BMI), body fat mass and percentage, waist circumference, blood pressure (SBP and DBP), serum glucose, lipids, insulin, and indices of insulin resistance (HOMA-IR) and sensitivity (QUICKY-IS) were compared between groups by analysis of covariance (ANCOVA), adjusting for country, gender and age. Non-normally distributed variables were previously log-transformed.

According to our data, adolescents with higher WBCC presented significantly higher weight $(P<0.01)$, BMI, body fat, waist circumference (all $P<0.001)$, DBP $(P<0.01)$, serum triglycerides $(P<0.001)$, total cholesterol/HDL-cholesterol and LDL/HDL-cholesterol ratios $(P<0.01)$, serum insulin and HOMA-IR index (both $P<0.001)$, and significantly lower values of serum HDL-cholesterol and QUICKY-IS index (both $P<0.001$ ).

These results support a significant positive association between WBCC and obesity and metabolic syndrome, and suggest that WBCC can be a useful marker to assess cardio-metabolic risk in European adolescents.

1. Ford ES (2003) Atherosclerosis 168, 351-358.

2. Nagasawa N, Tamakoshi K, Yatsuya H, et al. (2004) Circ J 68(10), 892-897.

3. Capuano V, Bambacaro A, Darminio T \& Mazzot G (2001) Ital Heart J 2(9), 685-689.

4. Wu CZ, Hsiao FC, Lin JD, et al. (2010) Acta Diabetol 47, 65-71.

5. Lee YJ, Shin YH, Kim JK, et al. (2010) Nutr Metab Cardiovasc Dis 20, 165-172. 